\title{
X-RAY ABSORPTION, NUCLEAR INFRARED EMISSION, AND DUST COVERING FACTORS OF AGNs: TESTING UNIFICATION SCHEMES
}

\author{
S. Mateos ${ }^{1}$, F. J. Carrera ${ }^{1}$, A. Alonso-Herrero ${ }^{1}$, A. Hernán-Caballero ${ }^{1}$, X. Barcons ${ }^{1}$, A. Asensio Ramos ${ }^{2,3}$, \\ M. G. Watson ${ }^{4}$, A. Blain ${ }^{4}$, A. Caccianiga ${ }^{5}$, L. Ballo ${ }^{5}$, V. Braito ${ }^{6}$, and C. Ramos Almeida ${ }^{2,3}$ \\ ${ }^{1}$ Instituto de Física de Cantabria (CSIC-Universidad de Cantabria), E-39005, Santander, Spain; mateos@ifca.unican.es \\ ${ }^{2}$ Instituto de Astrofísica de Canarias, E-38205, La Laguna, Tenerife, Spain \\ ${ }^{3}$ Departamento de Astrofísica, Universidad de La Laguna, E-38206, La Laguna, Tenerife, Spain \\ ${ }^{4}$ Physics and Astronomy, University of Leicester, University Road, Leicester LE1 7RH, UK \\ ${ }^{5}$ INAF-Osservatorio Astronomico di Brera, via Brera 28, I-20121 Milano, Italy \\ ${ }^{6}$ INAF-Osservatorio Astronomico di Brera, Via Bianchi 46, I-23807 Merate (LC), Italy \\ Received 2015 November 27; accepted 2016 January 21; published 2016 March 9
}

\begin{abstract}
We present the distributions of the geometrical covering factors of the dusty tori $\left(f_{2}\right)$ of active galactic nuclei (AGNs) using an X-ray selected complete sample of 227 AGNs drawn from the Bright Ultra-hard XMM-Newton Survey. The AGNs have $z$ from 0.05 to $1.7,2-10 \mathrm{keV}$ luminosities between $10^{42}$ and $10^{46} \mathrm{erg} \mathrm{s}^{-1}$, and Comptonthin X-ray absorption. Employing data from UKIDSS, 2MASS, and the Wide-field Infrared Survey Explorer in a previous work, we determined the rest-frame 1-20 $\mu \mathrm{m}$ continuum emission from the torus, which we model here with the clumpy torus models of Nenkova et al. Optically classified type 1 and type 2 AGNs are intrinsically different, with type 2 AGNs having, on average, tori with higher $f_{2}$ than type 1 AGNs. Nevertheless, $20 \%$ of type 1 AGNs have tori with large covering factors, while $23 \%-28 \%$ of type 2 AGNs have tori with small covering factors. Low $f_{2}$ are preferred at high AGN luminosities, as postulated by simple receding torus models, although for type 2 AGNs the effect is certainly small. $f_{2}$ increases with the X-ray column density, which implies that dust extinction and X-ray absorption take place in material that share an overall geometry and most likely belong to the same structure, the putative torus. Based on our results, the viewing angle, AGN luminosity, and also $f_{2}$ determine the optical appearance of an AGN and control the shape of the rest-frame $\sim 1-20 \mu$ m nuclear continuum emission. Thus, the torus geometrical covering factor is a key ingredient of unification schemes.
\end{abstract}

Key words: galaxies: active - galaxies: nuclei - infrared: galaxies - quasars: general

\section{INTRODUCTION}

The simplest standard unified models postulate that the diversity of observed properties of active galactic nuclei (AGNs) can be largely explained as a viewing angle effect and anisotropic nuclear obscuration (Antonucci 1993; Urry \& Padovani 1995). A key ingredient of these orientation-based models is an optically and geometrically thick toroidal structure located on tens of parsec scales that obscures the AGN nuclear region (accretion disk and X-ray corona) and the broad-line region from certain lines of sight. For the sake of simplicity we will refer to this structure as the "torus." Orientation-based unified models of tori with homogeneous dust distributions propose that AGNs are optically classified as type 1 if they are observed at low inclinations with respect to the axis of the torus. In this case the line of sight does not intercept the material in the torus and we have an unobscured view of the central engine. On the other hand, AGNs are optically classified as type 2 if they are observed at high inclinations where the material in the torus does intercept the line of sight obscuring the central engine (see Netzer 2015 for a recent review).

In recent years it has been realized that AGN luminosity had to be incorporated as a key ingredient of unified models in order to explain the observed decrease in the relative fraction of type 2 objects at high AGN luminosities. This trend, mainly detected in X-ray surveys, has been further confirmed by surveys at optical and infrared wavelengths (e.g., Hasinger et al. 2005; Simpson 2005; Della Ceca et al. 2008; Treister et al. 2008; Ebrero et al. 2009; Burlon et al. 2011; Ueda et al. 2014; Assef et al. 2015; Buchner et al. 2015; Lacy et al.
2015). To explain the scarcity of luminous type 2 AGNs, the "receding torus model" has been often invoked. This model postulates that the luminosity-dependence of the type 2 AGN fraction is directly associated with the geometry of the torus in the sense that the covering factor of the torus (the fraction of the sky as seen by the source obscured by dust) decreases with increasing AGN luminosity (Lawrence 1991). Unfortunately, it is not yet fully understood how the AGN accretion power can influence the physical extent of the torus, as there is substantial quantitative disagreement between published luminosity trends (e.g., Lawrence \& Elvis 2010; Sazonov et al. 2015).

The nuclear spectral energy distributions (SEDs) and midinfrared interferometric observations of nearby AGNs are modeled better with clumpy dusty tori, i.e., the obscuring material is not uniformly distributed inside the torus. In fact the material appears to be distributed in discrete, optically thick clumps (e.g., Alonso-Herrero et al. 2003; Tristram et al. 2007, 2009; Markowitz et al. 2014). X-ray spectral variability studies have also confirmed that the gas responsible for most of the X-ray absorption must be clumpy (e.g., Risaliti et al. 2009, 2011; Brenneman et al. 2013).

The clumpy nature of the dusty torus has very important implications for unification models, as the classification of an AGN turns out to be an orientation-dependent probability. This means that even if we observe from an AGN's equator, there is some probability of classifying it as type 1 , while a pole-on AGN could still be classified as type 2 if a single cloud intercepts the line of sight. Thus, while in the simplest orientation-based models with a smooth torus type 1 and type 2 AGNs should have tori sharing the same properties, in the 
framework of "clumpy unification" type 2 AGNs should have tori with geometrical covering factors higher, on average, than type 1 AGNs (for a recent review on this topic see Elitzur 2012). Recent analyses of the nuclear infrared emission of AGNs with radiative transfer models of clumpy tori indicate that this might indeed be the case (Ramos Almeida et al. 2011; Ichikawa et al. 2015). Unfortunately, until very recently such studies have been restricted to small samples of mostly nearby Seyfert galaxies and quasars (Mor et al. 2009; Nikutta et al. 2009; Ramos Almeida et al. 2009, 2011; Alonso-Herrero et al. 2011; Deo et al. 2011; Lira et al. 2013).

Thanks to the advent of the all-sky infrared survey conducted with the Wide Field Infrared Survey Explorer at 3.4, 4.6, 12, and $22 \mu \mathrm{m}$ (WISE; Wright et al. 2010) it is now possible to constrain the properties of the AGN tori, in particular its geometrical covering factor, in large, representative samples of objects spanning a broad range of both redshifts and AGN luminosities.

The aim of this study is to verify observationally the validity of unified schemes in the framework of clumpy torus models. To do so we have determined, for the first time, the distribution of covering factors of AGN tori using a large, uniformly selected, complete sample of AGNs. We have investigated whether type 1 and type 2 AGNs are indeed intrinsically different objects, as recently claimed in the literature, by comparing the distributions of covering factors of their tori. Finally we have determined the dependence (or lack of) of the torus covering factor on the line of sight absorption measured in X-rays and the AGN luminosity.

The 227 AGNs used in this study are drawn from the Bright Ultra-hard XMM-Newton Survey (BUXS; Mateos et al. 2012, 2015; hereafter M15). The objects have $z$ in the range 0.05-1.7, intrinsic (absorption-corrected) $2-10 \mathrm{keV}$ $\mathrm{X}$-ray luminosities between $10^{42}$ and $10^{46} \mathrm{erg} \mathrm{s}^{-1}$, and X-ray absorption in the Compton-thin regime. There are a number of reasons why we have chosen the BUXS survey to conduct this study: first, its large sample size and high spectroscopic identification completeness: BUXS is one of the largest (255 objects) complete flux-limited samples of bright AGNs selected at energies above $4.5 \mathrm{keV}$ with the XMM-Newton observatory. At the time of writing optical spectroscopic classifications and accurate redshifts are available for $98.4 \%$ of the objects; second, the rich set of multi-wavelength data available for all sources: good-quality XMM-Newton spectroscopy is available for the full sample, enabling accurate measurements of both the $\mathrm{X}$-ray absorption and intrinsic X-ray luminosities for all objects. Furthermore, 227 out of 233 AGNs with X-ray luminosities and redshifts in the chosen intervals have restframe 1-20 $\mu \mathrm{m}$ nuclear photometric SEDs associated with the emission from the AGN dusty torus from M15. Clearly, all these properties make our AGN sample ideally suited to draw robust statistical constraints on the properties of the dusty torus of AGN.

From all the radiative transfer codes available in the literature to model the infrared emission associated with clumpy tori (e.g., Nenkova et al. 2002, 2008a, 2008b, henceforth referred to collectively as N08; Dullemond \& van Bemmel 2005; Schartmann et al. 2008; Hönig et al. 2010; Stalevski et al. 2012; Siebenmorgen et al. 2015) we have chosen the N08 models, referred to as CLUMPY models, as they provide a good representation of the torus SED, and facilitate direct comparison with previous results in the literature (Mor et al. 2009; Nikutta et al. 2009; Ramos Almeida et al. 2009, 2011; Alonso-Herrero et al. 2011; Deo et al. 2011; Lira et al. 2013).

This paper is structured as follows. Section 2 describes the AGN sample used in this study. In Sections 3.1 and 3.2 we present the SED fitting techniques used to first isolate the emission associated with the torus and then to model it with the N08 models. In Section 3.3 we discuss our approach to determine the distributions of covering factors of AGN tori. In Section 4 our results are presented and discussed, while in Section 5 we summarize our main results. Throughout this paper, errors are at $68 \%$ confidence for a single parameter, and we assume $\Omega_{\mathrm{M}}=0.3, \Omega_{\Lambda}=0.7$, and $H_{0}=70 \mathrm{~km} \mathrm{~s}^{-1} \mathrm{Mpc}^{-1}$.

\section{AGN SAMPLE}

The AGNs in this study are drawn from the wide-angle Bright Ultra-hard XMM-Newton Survey (BUXS). BUXS is a complete flux-limited sample of 255 X-ray bright AGNs $\left(f_{4.5-10 \mathrm{keV}}>6 \times 10^{-14} \mathrm{erg} \mathrm{s}^{-1} \mathrm{~cm}^{-2}\right)$ detected at 4.5 to $10 \mathrm{keV}$ energies with the XMM-Newton European Photon Imaging Camera (EPIC)-pn (Strüder et al. 2001). The objects were selected at such high energies to reduce biases against highly absorbed AGNs as much as possible. The survey covers $44.43 \mathrm{deg}^{2}$ of the northern sky (galactic latitudes $|b|>20^{\circ}$ ) distributed over 381 XMM-Newton observations with good quality for serendipitous source detection (Mateos et al. 2008, 2012). For complete details on the survey design, sample selections and UV/optical spectroscopic identification and classification of the objects, see Mateos et al. (2012, 2015). At the time of writing the identification completeness is $98.4 \%$ (251 objects). Such a high identification rate guarantees that our study will not suffer from biases associated with optical identification incompleteness, which are more severe for highly obscured type 2 AGNs.

We have good-quality XMM-Newton spectra for all BUXS sources, which constrain directly both the line of sight restframe absorbing column densities and X-ray luminosities (see M15). Throughout this paper $L_{X}$ represents intrinsic, absorption-corrected luminosities in the rest-frame 2-10 keV band.

For the analysis presented here we selected the 233 nonblazar AGNs with $L_{X}>10^{42}$ and $z<1.7$. The luminosity cut was applied to reduce to a minimum the uncertainties associated with the determination of the infrared emission of the tori of our objects by increasing the contrast of the AGNs over the underlying emission from the AGN hosts (12 objects removed; see Section 3.1). The redshift cut was imposed to assure adequate wavelength sampling of the torus rest-frame continuum emission (six objects removed). Finally, we excluded five type $1 \mathrm{AGNs}$ and one type 2 AGN without detections with signal-to-noise-ratios $(\mathrm{S} / \mathrm{N})>2$ at all 3.4, 4.6, and $12 \mu \mathrm{m}$ in the final data release of WISE (AllWISE; Cutri et al. 2013). As the number of objects not detected with WISE above our selection threshold is rather small, and in addition, these objects span a broad range of $L_{\mathrm{X}}$ and $z$, removing them from the sample should not bias our results. All the above selection criteria left us with a sample of 227 AGNs with $L_{X}$ from $10^{42}$ to $10^{46} \mathrm{erg} \mathrm{s}^{-1}$ and $0.05<z<1.7$.

We classified our AGNs as type 1 if permitted and semiforbidden broad emission lines (line velocity widths $\gtrsim 1500 \mathrm{~km} \mathrm{~s}^{-1}$ ) were detected in their rest-frame UV/optical spectra (132 objects) and as type 2 if they showed narrow 
emission lines only (line velocity widths $<1500 \mathrm{~km} \mathrm{~s}^{-1} ; 75$ objects) or had a galaxy-like spectrum with no emission lines (3 objects). Due to the controversy regarding the nature of intermediate Seyfert types 1.8 and 1.9 as type 1 or type 2 AGN we kept such objects as a separate class (17 objects). ${ }^{7}$

\section{METHODOLOGY}

\subsection{Isolating the Torus Emission}

In M15 we determined infrared SEDs associated with the emission from the dusty torus for the AGNs in BUXS. To do so we conducted a thorough analysis of the rest-frame UV-toinfrared photometric SEDs to correct the cataloged infrared fluxes for any contamination associated with both the host galaxies and the direct emission from the AGN accretion disk. Our SEDs are based on data from the Sloan Digital Sky Survey (SDSS; Abazajian et al. 2009), the Two Micron All Sky Survey (2MASS; Jarrett et al. 2000; Cutri et al. 2003), the UKIRT Infrared Deep Sky Survey (UKIDSS; Lawrence et al. 2007), and WISE (Wright et al. 2010). To decompose the observed fluxes into AGN and galaxy emission we used the SED fitting tool SEd Analysis using BAyesian Statistics (SEABAS, Rovilos et al. 2014).

Very briefly, to model the emission from the accretion disk we used the type 1 quasar SED from Richards et al. (2006) at rest-frame wavelengths $\lambda<0.7 \mu \mathrm{m}$ and a power-law $\lambda f_{\lambda} \propto \lambda^{-1}$ at longer wavelengths. To redden the accretion disk we used the Gordon \& Clayton (1998) Small Magellanic Cloud extinction law at $\lambda<0.33 \mu \mathrm{m}$ and the Cardelli et al. (1989) Galactic extinction law at $\lambda>0.33 \mu \mathrm{m}$. In both cases we assumed $R_{V}=3.1$. To characterize the continuum emission from the AGN dusty torus we used the Seyfert 1 and the two Seyfert 2 templates corresponding to rest-frame X-ray absorbing column densities $N_{H}<10^{24} \mathrm{~cm}^{-2}$ from Silva et al. (2004). Finally, to reproduce the emission from the stellar population of the AGN hosts at rest-frame optical-near-infrared wavelengths we used a library of 75 stellar templates from Bruzual \& Charlot (2003). The templates have solar metallicity and a Chabrier initial mass function (Chabrier 2003) and were generated using 10 exponentially decaying star formation histories with characteristic times $\tau=0.1-30$ Gyr and a model with constant star formation, and a set of ages in the range 0.1-13 Gyr. To redden the stellar templates we used the Calzetti et al. (2000) dust extinction law. An example of the SED decomposition analysis is illustrated in Figure 1. It is important to highlight that we have adopted the same templates and SED decomposition procedure to isolate the AGN torus emission of all sample objects.

In M15 we demonstrated that stellar contamination of cataloged fluxes in the infrared regime at rest-frame wavelengths shorter than $\sim 6 \mu \mathrm{m}$ is significant, especially for type 2 objects. Only for type 1 objects with $L_{X}>10^{44} \mathrm{erg} \mathrm{s}^{-1}$ do the AGNs outshine the host galaxy in the infrared band. The tight correlation found between rest-frame $6 \mu \mathrm{m}$ luminosities, corrected for contamination from the accretion disk and AGN hosts, and 2-10 keV intrinsic (absorption-corrected) luminosities, supports the hypothesis that the infrared SEDs determined from our decomposition analysis are associated with dust

\footnotetext{
7 Objects of intermediate Seyfert type 1.9 can be identified up to $z \sim 0.2-0.4$, depending on whether or not the $\mathrm{H} \alpha$ emission line is outside the observable wavelength range of our spectroscopic data.

8 http://astro.dur.ac.uk/ erovilos/SEABASs/
}

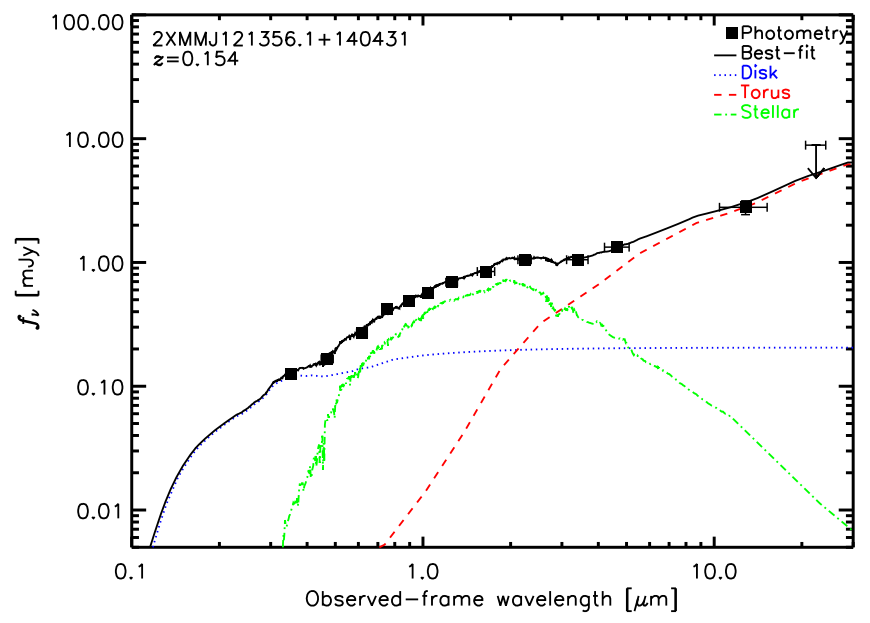

Figure 1. Example of the SED decomposition analysis used in M15 to isolate the AGN dusty torus emission (see Section 3.1 for details). The example corresponds to an AGN optically classified as type 1. Filled squares are the cataloged photometry. The dotted, dashed, and dot-dashed lines correspond to the accretion disk, torus (Seyfert 1 template from Silva et al. 2004), and host galaxy emission, respectively. The solid line is the best-fit model. We note that in the SED decomposition analysis we treated all $22 \mu \mathrm{m}$ detections (168 in total) as upper limits (see M15 for details).

heated by the intense radiation field of the AGN. This dust is most likely located in the putative torus on parsec scales. Hereafter, nuclear infrared SEDs will refer to the emission from the torus.

We focus our analysis here on rest-frame wavelengths longer than $1 \mu \mathrm{m}$ since this is the spectral region where the AGN torus emits the bulk of its radiation. Although in M15 we demonstrate that, at the luminosities of our AGNs, contamination due to star formation at these wavelengths should be negligible, to minimize such effects we have treated the WISE $22 \mu \mathrm{m}$ fluxes of all 12 objects with $L_{\mathrm{X}}<5 \times 10^{42} \mathrm{erg} \mathrm{s}^{-1}$ (five type $1 \mathrm{AGNs}$, six type 2 AGNs, and one Sy1.9), whether detected or not at these wavelengths, as upper limits. Nevertheless, we have checked that this assumption does not affect our main results.

\subsection{SED Fitting with CLUMPY Models}

To describe the nuclear infrared emission of our objects we have used the radiative transfer models by N08. In these models the material obscuring the AGN nuclear region is treated as a medium with a toroidal shape, where the dust and gas are distributed in high-density clumps inside it. The angular distribution of clouds has no sharp cutoff boundary and is described as a Gaussian of width $\sigma$,

$$
N_{\mathrm{T}}(\beta)=N_{0} e^{\left(-\beta^{2} / \sigma^{2}\right)},
$$

where $N_{\mathrm{T}}$ is the line of sight number of clouds, $\beta=\pi / 2-i$ is the inclination angle of the torus equatorial plane with respect to the line of sight and $i$ is the viewing angle from the torus axis. $N_{0}$ represents the mean number of clouds along radial equatorial rays. In the N08 models the radial distribution of clouds is parameterized as a power law, $N(r) \propto r^{-q}$, where $N$ is the number of clouds and $q$ is the power-law index. The torus radial thickness $(Y)$ is defined as the ratio of the outer $\left(R_{\mathrm{o}}\right)$ to inner $\left(R_{\mathrm{d}}\right)$ radius of the distribution of clouds, where $R_{\mathrm{d}}$ is set by the AGN luminosity and the dust sublimation temperature 
Table 1

Parameters of the N08 CLUMPY Torus Models and Range of Values Used in this Work

\begin{tabular}{ll}
\hline \hline Parameter & Range \\
\hline Torus angular width $(\sigma)$ & {$\left[15^{\circ}-70^{\circ}\right]$} \\
Torus radial thickness $(Y)$ & {$[5-30]$} \\
Mean number of clouds along equatorial rays $\left(N_{0}\right)$ & {$[1-15]$} \\
Index of the radial distribution of clouds $(q)$ & {$[0-3]$} \\
Single cloud optical depth $\left(\tau_{\mathrm{V}}\right)$ & {$[5-150]$} \\
Viewing angle $(i)$ & {$\left[0^{\circ}-90^{\circ}\right]$} \\
\hline
\end{tabular}

( $\sim 1500 \mathrm{~K}$ in the model; Barvainis 1987). All clouds are assumed to be optically thick and with the same optical depth, defined in the $V$-band at $5500 \AA$. The model assumes a standard cold oxygen-rich interstellar medium dust extinction law (Ossenkopf et al. 1992). In addition to the parameters defining the geometry of the torus and the properties of the clouds, the scaling factor required to match the fluxes from the best-fit model to the observed values in the SEDs can be used as proxy for the AGN bolometric luminosity (see Nenkova et al. 2008b; Alonso-Herrero et al. 2011). We refer the reader to N08 for further details on CLUMPY models.

The online database of CLUMPY consists of more than $10^{6}$ models based on a narrow grid for each torus parameter. ${ }^{9}$ With such a fine grid we will not be able to distinguish between torus models based on different combinations of parameters but with differences in the continuum shape smaller than our SED photometric uncertainties. In that sense we can say that in our analysis there is a strong degeneracy in the parameters of CLUMPY models. To best deal with this issue we have conducted the SED fits using the code BayesCLUMPY from Asensio Ramos \& Ramos Almeida (2009). This code has been especially developed to analyze the emission of AGN tori with CLUMPY models using a Bayesian inference approach. BayesCLUMPY uses a Metropolis-Hastings Markov Chain Monte Carlo (MCMC) sampling technique to determine posterior distributions for each parameter. To ensure the continuity of parameters, BayesCLUMPY also interpolates in the original database of models. In our analysis we have used truncated uniform prior distributions for all parameters in the ranges listed in Table 1.

\subsection{Covering Factors of AGN Tori}

In torus models with a clumpy distribution of dust the UV/ optical appearance of an AGN depends on the viewing angle and the probability of intercepting a dusty cloud along our line of sight. Thus, the geometrical covering factor of the torus should play a fundamental role in the optical classification of AGNs: the smaller the covering factor, the higher the probability of a direct view of the AGN nuclear region. Assuming that all individual clouds are optically thick, as in the N08 models, the probability that light from the AGN at an angle $\beta$ will escape unaffected from the torus has the form

$$
P_{\mathrm{esc}}(\beta)=e^{-N_{0} e^{\left(-\beta^{2} / \sigma^{2}\right)}},
$$

The geometrical covering factor of the torus representing the fraction of the sky obscuring the AGN nuclear region, $f_{2}$, is

\footnotetext{
9 http://www.pa.uky.edu/clumpy/
}

defined as

$$
f_{2}=1-\int_{0}^{\pi / 2} P_{\mathrm{esc}}(\beta) \cos (\beta) d \beta,
$$

where $P_{\text {esc }}$ is integrated over all angles (Mor et al. 2009). As $f_{2}$ is independent of the inclination angle, it represents the true intrinsic fraction of optically obscured type 2 objects in the entire AGN population.

Figure 2 shows three examples of the typical nuclear infrared SEDs used in our study and the SED fitting results obtained with BayesCLUMPY. The insets show the normalized posterior distributions of $f_{2}$ derived from the fits. To obtain the distribution of $f_{2}$ for a sample of objects fully taking into account the uncertainties from the fits we first concatenated together the individual arrays of values of $f_{2}$ returned from the MCMC analysis for each object and then we computed the probability distribution of the combined array of values of $f_{2}{ }^{10}$ To compare different distributions of $f_{2}$ we have used the twosample Kolmogorov-Smirnov (KS) test and Monte Carlo simulations to determine the probability of rejecting the null hypothesis that the two samples are drawn from the same parent population. Each time we ran our simulations we used bootstrap to randomly select $10^{5}$ times the sample objects used to determine the distributions of $f_{2}$.

For illustration purposes, throughout this paper we have used a bin size of 0.03 to represent the distributions of $f_{2}$, but we stress that all computations are based on the arrays of values of $f_{2}$. All distributions are normalized to have an area of one under the curve.

\section{RESULTS}

As indicated before, we have used the N08 models to reproduce the nuclear infrared emission associated with the dusty tori of AGN and to determine their dust covering factors. Therefore, the results inferred from our SED fits should be considered in the framework of these models.

\section{1. $f_{2}$ versus Optical Class}

Figure 3 shows the distribution of $f_{2}$ for our full sample of AGNs. It is evident that type 1 and type 2 AGNs have significantly different distributions of $f_{2}$, in the sense that type 2 AGNs overall have tori with higher covering factors than type 1 AGNs. Based on the KS test and our simulation analysis we can reject the null hypothesis that the two samples are drawn from the same parent population with a confidence level higher than $99.99 \%$. Nevertheless, we find that there is a large overlap between the distributions of $f_{2}$ for type 1 and type 2 AGNs, in good agreement with previous studies based on high-spatial resolution nuclear infrared photometric data and/or midinfrared spectroscopic data for small samples of local Seyferts and PG quasars (e.g., Mor et al. 2009; Ramos Almeida et al. 2009, 2011; Alonso-Herrero et al. 2011; Ichikawa et al. 2015).

The distributions of $f_{2}$ that we find for the two AGN populations are significantly broader than claimed in the above studies. As we will see in the following sections, such apparent discrepancies are not associated with higher uncertainties in our

\footnotetext{
${ }^{10}$ While we believe that the methodology applied is reliable, a more coherent way to infer the global distribution of the covering factor would have been to use a hierarchical Bayesian model. Nevertheless, such an approach would have entailed the typical shrinkage of hierarchical modeling.
} 

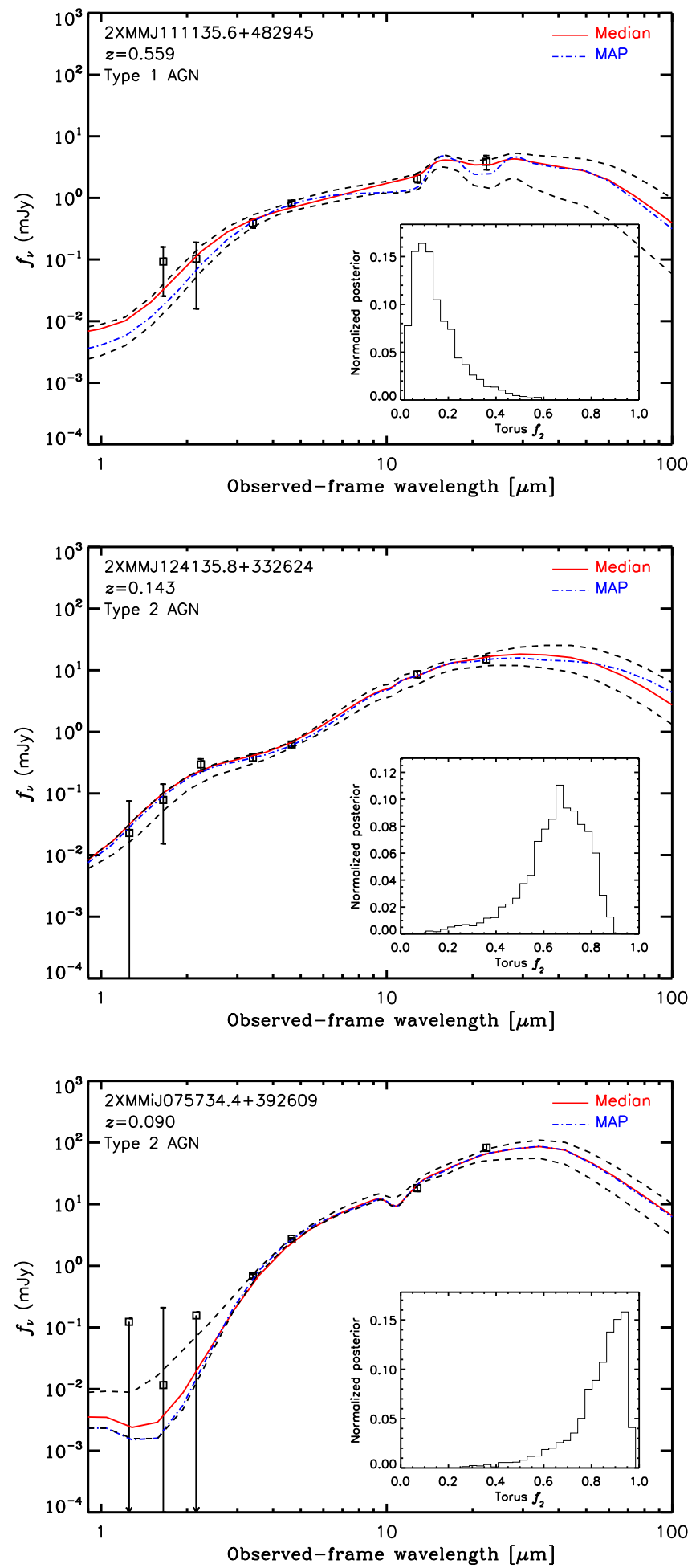

Figure 2. Examples of the SEDs of AGN dusty tori used in our study (open symbols and error bars). Vertical arrows indicate upper limits. The SEDs have been corrected for the emission associated with both the accretion disk and the AGN host galaxies. The solid and dot-dashed lines correspond to the torus models described with the maximum (mode-MAP) and median values of the posterior probability distributions of the parameters returned by the BayesCLUMPY fits, respectively. The dashed lines indicate the range of models enclosing a $68 \%$ probability. The inset plots show the normalized posterior distributions of $f_{2}$ from the fits.

analysis compared to previous studies but to type 1 (type 2) AGNs having rather large (small) torus covering factors. Since

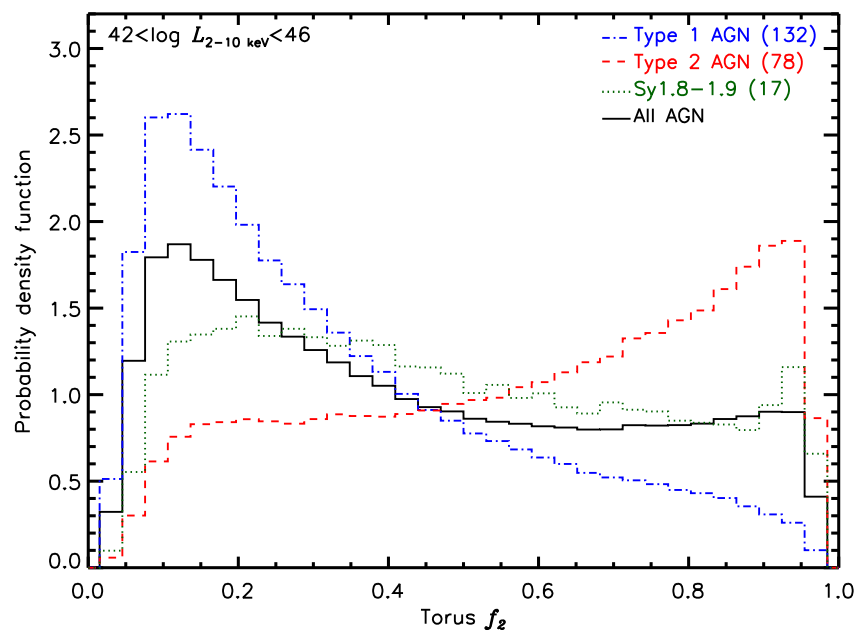

Figure 3. Distributions of the covering factors of AGN tori calculated for the full sample of objects.

such objects are rare, sampling them requires studies of large and complete samples of AGNs, such as ours. For example, as we will see in Section 4.4.1, if we use $f_{2}=0.5$ as a threshold to separate AGN tori with low and high covering factors, we find that 26 out of 132 type 1 AGNs have tori with high covering factors, while 22 out of 78 type 2 AGNs have tori with low covering factors.

As indicated before, we stress that we have used the same SED decomposition procedure to isolate the AGN torus emission of all sample objects. Our SED fits with BayesCLUMPY take into account both the quality of the cataloged infrared photometric data, which is similar among our type 1 and type 2 AGNs, and the uncertainties from the SED decomposition analysis. We have also checked that the distributions of $f_{2}$ for type 1 AGN have widths, based on the 16th and 84th percentiles, that are indistinguishable from those of type 2 AGN. All this guarantees that any differences in the torus properties among AGNs with different optical spectroscopic classifications reported here are genuine and not an artifact of either the SED decomposition analysis or the quality of the infrared data.

Interestingly, we find that AGNs classified as Sy1.8-1.9 have a rather flat distribution of $f_{2}$ that is significantly different from those of type 1 and type 2 AGNs. Thus, from the infrared point of view, we find that it is highly unlikely that all Sy 1.8-1.9 are simply ordinary type 1 AGNs caught in a low flux state during the UV/optical spectroscopic observations, as significant differences exist between the properties of the tori of these two AGN classes. A KS test returns probabilities of $99.78 \%$ and $97.98 \%$ for rejecting the null hypothesis that the distributions of $f_{2}$ for Sy 1.8-1.9 and those obtained for type 1 and type 2 AGN are identical. Therefore, alternative processes must play a role. For example, our finding that 12 out of the 17 intermediate-type objects are absorbed in X-rays (see Section 4.2) favors a scenario where most of our Sy1.8-1.9 objects have broad-line regions reddened by optically thin dust located either in the torus or on physical scales of the narrowline region or the host galaxies (e.g., Alonso-Herrero et al. 2011).

We note that, if some high- $z$ Sy1.8-1.9 objects are still present in our sample of type 2 AGNs, the effect would be to reduce the differences between the $f_{2}$ distributions of type 1 and 
type 2 AGNs. Clearly, this would not change our results since we have already found that we can reject the hypothesis that the distributions of $f_{2}$ for type 1 and type 2 AGNs are drawn from the same parent population with a confidence level higher than $99.99 \%$.

Finally, we have used the results from our SED decomposition analysis to compare the distributions of $f_{2}$ for all objects with detected UV/optical broad emission lines (132 type 1 AGN and 17 Sy1.8-1.9s) with low and high extinction toward their accretion disk. To separate the objects we have used an extinction of $E(B-V)=0.32$, or $A_{V} \sim 1 \mathrm{mag}$ assuming a Galactic standard conversion. Such a value has often been used in the literature to identify moderately reddened type 1 AGNs (e.g., Urrutia et al. 2012; Lacy et al. 2013 and references therein). Based on the chosen extinction threshold, 13 out of 132 type 1 AGNs and 10 out of 17 Sy1.8-1.9s are classified as moderately reddened objects $(E(B-V)$ in the range $0.32-0.65$; see M15 for details). We find that, as expected, higher $f_{2}$ are preferred in reddened broad-line AGN. According to the KS test we can reject the null hypothesis that the $f_{2}$ distributions of the two samples (reddened and unreddened broad-line AGNs) are drawn from the same parent population with a $99.3 \%$ confidence level.

Based on the results presented in this section we can conclude that, type 1 , type 2 , and probably also intermediatetype AGNs are on average intrinsically different, as has been previously reported in the literature (e.g., Ramos Almeida et al. 2011).

\section{2. $f_{2}$ versus $X$-Ray Absorption}

The discovery that the UV/optical spectroscopic classifications of AGNs correlate well with the absorption properties measured in X-rays has provided strong observational evidence favoring standard orientation-based unified models. Nevertheless, it is well known that AGNs exhibit a large range of dust-to-gas ratios and that for a non-negligible fraction of objects, gas absorption in X-rays and dust extinction in the UVto-infrared spectral band are not always detected together (e.g., Mainieri et al. 2002; Mateos et al. 2005a, 2005b, 2010; Tozzi et al. 2006; Garcet et al. 2007; Winter et al. 2009; Corral et al. 2011; Page et al. 2011; Scott et al. 2011; González-Martín et al. 2014; Merloni et al. 2014).

To investigate whether a physical or geometrical connection exists between the material responsible for the X-ray absorption and UV-to-infrared obscuration, we have computed the distributions of $f_{2}$ for AGNs with different levels of X-ray absorption. To have a good representation of both type 1 and type 2 AGNs across the full range of measured X-ray column densities, we have divided the sample in four different bins: $N_{H}<4 \times 10^{21} \mathrm{~cm}^{-2}$ (henceforth X-ray unabsorbed) $4 \times 10^{21}<N_{H}<10^{23} \mathrm{~cm}^{-2}, \quad 10^{23}<N_{H}<4 \times 10^{23} \mathrm{~cm}^{-2}$ and $4 \times 10^{23}<N_{H}<1.4 \times 10^{24} \mathrm{~cm}^{-2}$. Figure 4 summarizes the results of this analysis. Although in Figure 4 we show the distributions of $f_{2}$ for objects classified as Sy1.8-1.9 for completeness, we do not use them in the analysis presented in this section, as we are clearly limited by small number statistics. Nevertheless, our results suggest that the distribution of $f_{2}$ is rather flat for both X-ray unabsorbed and absorbed Sy1.8-1.9s.

We note that none of our X-ray selected sources have a bestfit X-ray column density in the Compton-thick regime. Nevertheless, considering the uncertainties in $N_{H}$, we cannot rule out unambiguously Compton-thick absorption in five type 2 AGNs (all five sources belong to the $4 \times 10^{23}<N_{H}<1.4 \times 10^{24} \mathrm{~cm}^{-2}$ bin). As an independent test, we have determined the $L_{\mathrm{X}}^{\mathrm{obs}} / L_{6} \mu \mathrm{m}$ luminosity ratio for these objects where $L_{\mathrm{X}}^{\mathrm{obs}}$ are observed (i.e., not corrected for intrinsic absorption) rest-frame $2-10 \mathrm{keV}$ luminosities and $L_{6 \mu \mathrm{m}}$ are the monochromatic luminosities of the torus emission at rest-frame $6 \mu \mathrm{m}$. The later have been shown to be a good proxy for the AGN intrinsic power (Lutz et al. 2004; Ramos Almeida et al. 2007; Fiore et al. 2009; Georgantopoulos et al. 2011; Mateos et al. 2015; Stern 2015). Based on the relationship between $L_{X}$ and $L_{6} \mu \mathrm{m}$ from M15 we find that, in all five cases, the $L_{\mathrm{X}}^{\mathrm{obs}} / L_{6 \mu \mathrm{m}}$ ratio is consistent with Compton-thin absorption. Finally, we have used a column density of $4 \times 10^{21} \mathrm{~cm}^{-2}$ to separate unabsorbed and absorbed AGNs. Assuming a Galactic standard dust-to-gas ratio, an $N_{H}$ of $4 \times 10^{21} \mathrm{~cm}^{-2}$ corresponds to $A_{V} \sim 2 \mathrm{mag}$, or $E(B-V) \sim 0.65$, the extinction level that separates optical type 1 from type 2 AGNs (Caccianiga et al. 2008; Merloni et al. 2014).

Interestingly we find that, type 1 and type 2 AGNs with similar levels of X-ray absorption have significantly different distributions of torus geometrical covering factors. This result also holds for X-ray unabsorbed objects (Figure 4 top left). We can reject the null hypothesis that the distributions of $f_{2}$ for X-ray unabsorbed type 1 and type 2 AGNs are drawn from the same parent population with a confidence higher that $99.99 \%$. Clearly, intrinsic differences exist among the torus properties of these two groups of objects. Thus, although the host galaxies could totally outshine the AGN emission in the optical band in some objects (e.g., Moran et al. 2002; Severgnini et al. 2003; Page et al. 2006), this cannot be the sole factor for determining the optical appearance of X-ray unabsorbed type 2 objects (Panessa \& Bassani 2002; Bianchi et al. 2008, 2012; Panessa et al. 2009; Gallo et al. 2013). Indeed only 4 out of 10 X-ray unabsorbed type 2 AGNs in our sample have $L_{X}<10^{43} \mathrm{erg} \mathrm{s}^{-1}$, where host galaxy dilution can be an important effect (Caccianiga et al. 2007).

The distributions of $f_{2}$ for X-ray unabsorbed type 1 and type 2 AGNs (Figure 4 top left) are largely indistinguishable from those of absorbed AGNs with $N_{H}$ in the range $4 \times 10^{21} \mathrm{~cm}^{-2}<N_{H}<10^{23} \mathrm{~cm}^{-2}$ (Figure 4 top right). This suggests that, up to column densities of $\sim 10^{23} \mathrm{~cm}^{-2}$, there is no significant correlation between $f_{2}$ and $N_{H}$. Nevertheless, at $N_{H}<10^{23} \mathrm{~cm}^{-2}$ gas and dust in the AGN hosts might be contaminating some of our measurements, especially for type 2 AGNs (e.g., Alonso-Herrero et al. 2003; Guainazzi et al. 2005; Goulding et al. 2012). Therefore we focus our attention on objects with column densities $N_{H}>10^{23} \mathrm{~cm}^{-2}$, as such high column densities should be associated with the torus. ${ }^{11}$ As we only have three type 1 objects with $N_{H}>10^{23} \mathrm{~cm}^{-2}$ in BUXS the distribution of $f_{2}$ for such objects may not be representative of the overall population of highly absorbed type 1 AGNs. Thus, in what follows we restrict our discussion to type 2 objects. Nevertheless, we note that, based on the KS test and our simulation analysis, we cannot reject the null

\footnotetext{
11 Typical optical extinctions associated with galactic dust lanes are $A_{V} \sim 0.5-1.5 \mathrm{mag}$. Such a level of extinction corresponds to gas column densities of $N_{\mathrm{H}}<10^{23} \mathrm{~cm}^{-2}$ for gas-to-dust ratios typical of nearby AGNs (Maiolino et al. 2001). For example, the column density toward the Galactic Center associated with molecular gas is $N_{\mathrm{H}} \sim$ a few $\times 10^{22} \mathrm{~cm}^{-2}$ (Sanders et al. 1984).
} 

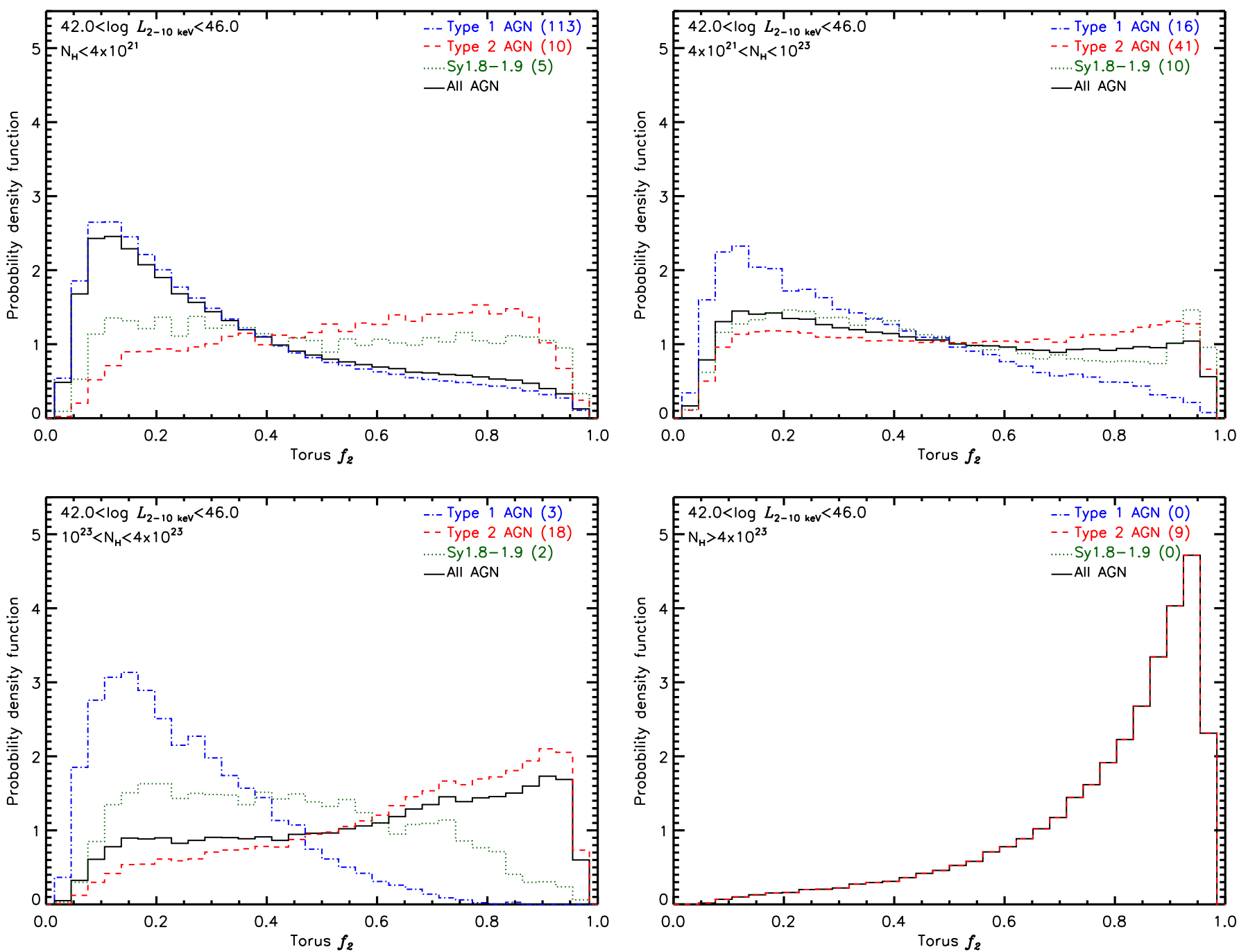

Figure 4. Dependence of the distribution of dust covering factors of AGN tori on the line of sight X-ray absorption.

hypothesis that the distributions of $f_{2}$ for unabsorbed, mildly absorbed $\left(4 \times 10^{21} \mathrm{~cm}^{-2}<N_{H}<10^{23} \mathrm{~cm}^{-2}\right)$, and highly absorbed $\left(10^{23} \mathrm{~cm}^{-2}<N_{H}<4 \times 10^{23} \mathrm{~cm}^{-2}\right)$ type 1 AGNs are drawn from the same parent population. We find that the covering factor of a typical type 2 AGN torus increases with $N_{H}$ (Figure 4 bottom plots). This effect becomes more pronounced at column densities approaching the Compton-thick regime.

Our analysis demonstrates that, not only do AGNs with different optical classifications have, on average, tori with different covering factors, but also the most highly absorbed type 2 AGNs have the highest covering factors of nuclear dust. Since all sources with absorbing column densities $N_{H}>4 \times 10^{23} \mathrm{~cm}^{-2}$ have remarkably similar distributions of $f_{2}$ it is highly unlikely that statistical fluctuations associated with the small sample size affect our results. Such, high dust covering factors seem to be a common property of the most absorbed Compton-thin type 2 AGNs (but see also Silva et al. 2004).

Interestingly, Ricci et al. (2011) found that type 2 AGNs with column densities in the range $10^{23} \mathrm{~cm}^{-2} \leqslant N_{H}<10^{24} \mathrm{~cm}^{-2}$ have a stronger X-ray reflection component than both type 1 and type 2 AGNs with $N_{H}<10^{23} \mathrm{~cm}^{-2}$. If the material in the torus is the main $\mathrm{X}$-ray reflector, these results are consistent with a scenario where the covering factor of the torus is higher in the most highly absorbed Compton-thin type 2 AGNs. This is supported by our findings.

$\mathrm{X}$-ray spectral variability studies have shown that a large fraction of the X-ray absorbing cold gas must be located at the physical scales of the broad-line region, probably in dust-free clouds in the innermost part of the torus, inside the dust sublimation radius (e.g., Elitzur 2008; Risaliti et al. 2009, 2011; Bianchi et al. 2012; Markowitz et al. 2014, and references therein). Our study supports these results, as the relationship between $f_{2}$ and $N_{H}$ that we find implies that the dust and most of the X-ray absorbing gas are at least geometrically related and plausibly belong to the same structure, the putative torus.

\section{3. $f_{2}$ versus AGN Luminosity}

Numerous works in the literature find that the relative fraction of type 2 AGNs decreases with increasing AGN luminosity (e.g., Hasinger et al. 2005; Della Ceca et al. 2008; Treister et al. 2008; Ebrero et al. 2009; Burlon et al. 2011; Ueda et al. 2014; Assef et al. 2015; Buchner et al. 2015; Lacy et al. 2015). These results have often been interpreted in the framework of the "receding torus" model. According to this model the radius at which the dust sublimates increases with 

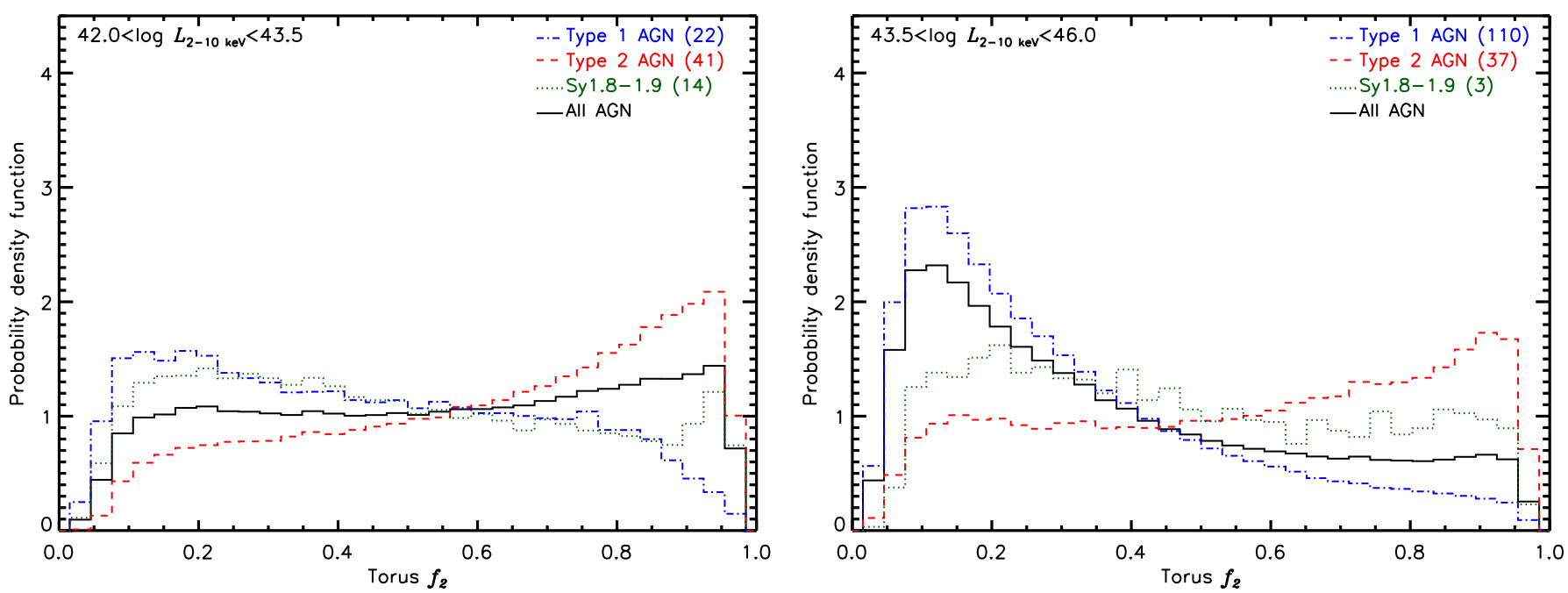

Figure 5. Dependence of the distribution of dust covering factors of AGN tori on the X-ray luminosity.

AGN luminosity, resulting in an increase of the opening angle of the torus and a decrease of its geometrical covering factor (Lawrence 1991). The end result is that the probability of finding an AGN as an optical type 2 is lower at high AGN luminosities.

To investigate whether we find any evidence supporting a scenario where the AGN radiation field can affect the torus properties, we have determined the distributions of $f_{2}$ in two luminosity bins. The results are illustrated in Figure 5. We clearly see that low covering factors are preferred in type 1 AGNs at high AGN luminosities. Although a similar trend is detected in type 2 objects, it is much less significant. This is somewhat expected, as a decrease in the torus covering factor reduces the probability of identifying an AGN as optical type 2. We have compared the distributions of $f_{2}$ obtained at low and high AGN luminosities for the same class of objects using the KS test. We can reject the null hypothesis that the two samples are drawn from the same parent population only for type 1 AGNs with a significance of $99.91 \%$.

Our sample of type 1 AGNs reaches $z \sim 1.7$ while all type 2 AGNs have $z \lesssim 1$. To avoid comparing objects at different evolutionary stages, we have also determined the distributions of $f_{2}$ in our two luminosity bins using type 1 and type 2 objects at $z<1$. Our results do not change. We note that we reach the same conclusion, adopting even lower redshift limits. This shows that although the rest-frame infrared spectral regions sampled with our photometric data vary with the objects' redshift, this has no effect on the $f_{2}$ distributions presented in Figure 5. Therefore, we can conclude that our results are robust and they are consistent with a decrease of the covering factor of AGN tori with increasing AGN luminosity, although for X-rayselected type 2 AGNs, the effect is modest. A detailed investigation of whether the detected changes of $f_{2}$ with luminosity are strong enough to explain the observed scarcity of type 2 AGNs at high luminosities will be presented in a forthcoming paper.

\subsection{Nuclear Infrared Continuum Emission}

\subsubsection{Dependence on $f_{2}$}

It is reasonable to expect that the properties of the nuclear infrared continuum emission of AGNs, in particular the broadband continuum shape, might depend directly on the covering factor of the torus. To investigate this issue we show in Figure 6 (top plots) a compilation of all nuclear infrared SEDs of our AGNs. ${ }^{12}$ As we are only interested in examining the continuum shape, we have normalized all SEDs at restframe $6 \mu \mathrm{m}$ to facilitate the comparison. To do so we have used linear interpolation in log-log space. We stress that we are only interested in the emission associated with the dusty torus, hence as indicated before, our nuclear infrared SEDs have been corrected for any contamination from the extrapolated accretion disk emission, and of course, the host galaxy.

The first result from Figure 6 is that there is a large range of torus-only infrared continuum shapes for both type 1 and type 2 AGNs. Clearly, at rest-frame wavelengths shorter than $\sim 20 \mu \mathrm{m}$ there is no canonical infrared slope for either AGN class (for similar results see also Alonso-Herrero et al. 2003; Lira et al. 2013). More importantly, we find that type 1 and type 2 AGNs show a similar range of infrared continuum slopes, although on average type 2 AGNs have steeper SEDs.

To investigate the role of the torus covering factor we have compared the SEDs of objects with tori with low $\left(f_{2}<0.5\right)$ and high $\left(f_{2}>0.5\right)$ covering factors, respectively. To assign the objects to the $f_{2}<0.5$ or $f_{2}>0.5$ class we have used the median values of the posterior distributions of $f_{2}$ obtained with the BayesCLUMPY SED fits for each source. We then moved all individual SEDs to rest-frame wavelengths and distributed the photometric data points into a common wavelength grid. The bins were defined to have at least 13 points and a minimum size of $0.01 \mu \mathrm{m}$. We have used the Astronomy Survival Analysis package (ASURV; Isobe et al. 1986) to determine the median flux values in each bin, taking into account both detections and upper limits. To determine the errors of the median SEDs while fully taking into account both the dispersion and errors in the individual fluxes, we used Monte Carlo simulations. For each photometric point, if it was an upper limit, we kept the values unchanged, while for detections we generated random numbers using Gaussian distributions of mean and sigma, the flux measurements, and their

\footnotetext{
12 The upper limits indicate cases where, based on our SED decomposition analysis, the AGN accretion disk and host galaxy emission account for the full observed flux. In such cases the upper limit is the combined error of the fluxes associated with the accretion disk and host galaxy emission.
} 

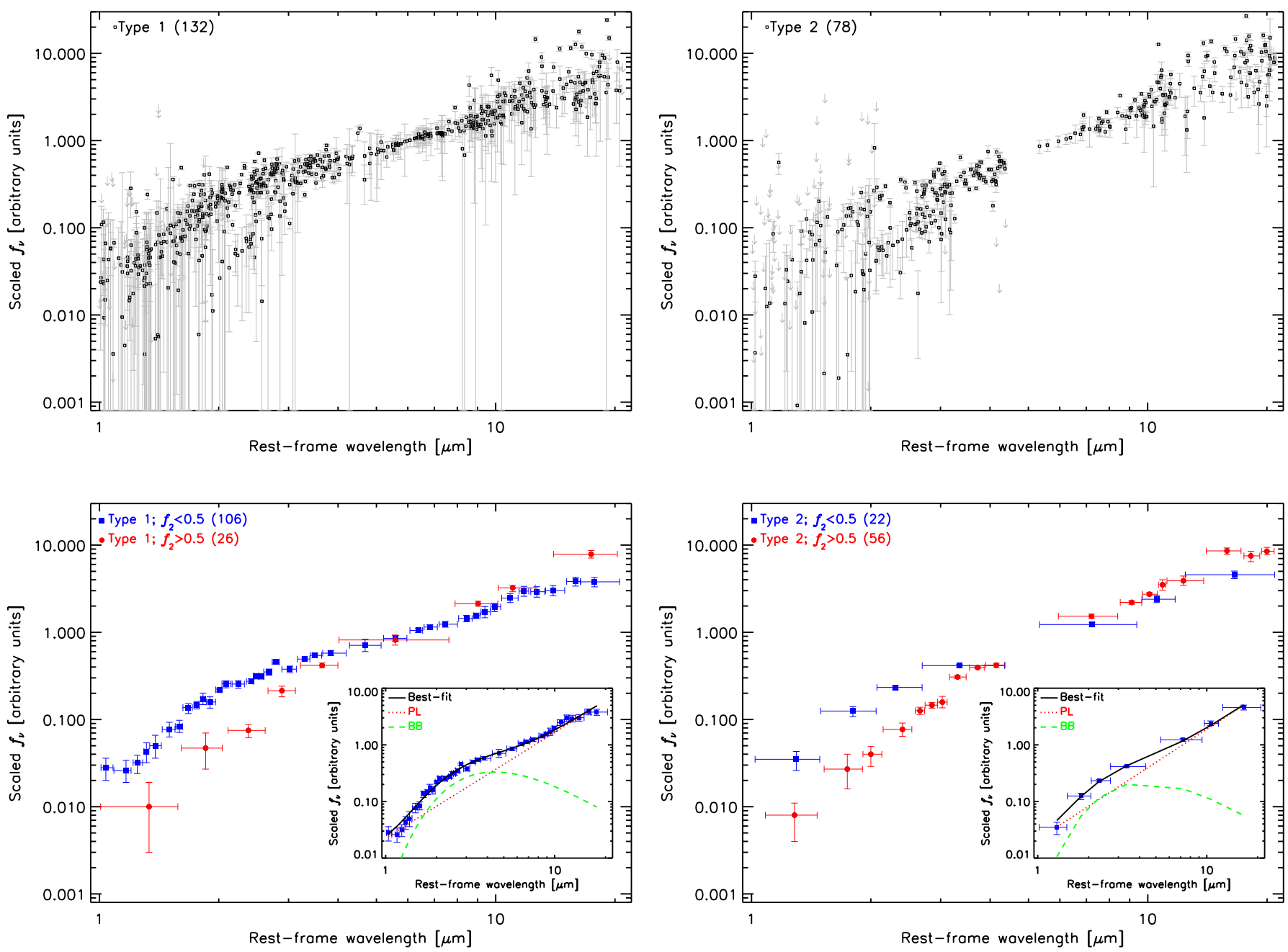

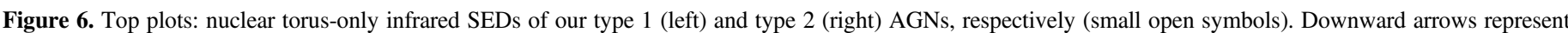

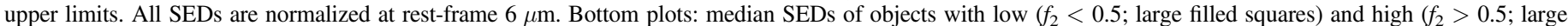

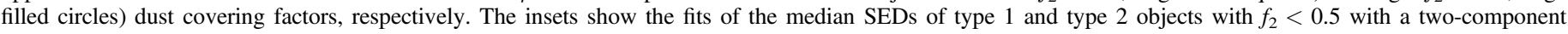

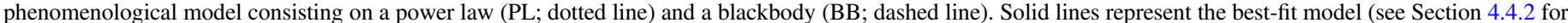
details).

corresponding uncertainties, respectively. In cases where the simulated flux values were lower than zero, we replaced them with the corresponding flux uncertainties and treated them as upper limits. We repeated the Monte Carlo exercise $10^{4}$ times, each time calculating median fluxes in the bins using the ASURV package, as we did for the real data. We then determined the uncertainties in our median SED fluxes using the 16th and 84th percentiles (68\% enclosed, equivalent to $1 \sigma$ ) of the distributions of simulated flux values on each bin. The results of this analysis are illustrated in Figure 6 (bottom plots).

We clearly see that AGNs with high $f_{2}$ have significantly redder SEDs on average than those with low $f_{2}$. We find the same result for both type 1 and type 2 objects. As indicated in Table 2, type 1 (type 2) AGNs with tori with low covering factors are on average about 5 (3) times more luminous than those with high covering factors. The observed differences in their nuclear infrared continuum emission could be a manifestation of the decrease of $f_{2}$ with AGN luminosity (see Section 4.3).

Interestingly, we find that $20 \%$ of type 1 AGNs have tori with large, $f_{2}>0.5$, covering factors, while $\sim 28 \%$ of type 2 AGNs have tori with small, $f_{2}<0.5$, covering factors. As pointed out in Section 2, Seyfert 1.9 objects can only be identified up to $z \lesssim 0.4$ hence, some might still be present in our sample of type 2 AGNs. To evaluate whether this has any impact on our results we have determined the fraction of type 2 AGNs at $z<0.3$ that have tori with $f_{2}<0.5$, finding a value of $22.7 \%$ (10 out of 44 objects). We can safely conclude that $\sim 23 \%-28 \%$ of type 2 AGNs have tori with small, $f_{2}<0.5$, covering factors.

To investigate whether differences exist in the continuum emission of AGN tori as a function of $f_{2}$ across the full range of wavelengths sampled, we have determined the spectral index that best describes the broadband continuum emission of AGN tori at rest-frame $5-20 \mu \mathrm{m}$. We have used a phenomenological model consisting of a simple power law ${ }^{13}$ that provides a good description of the data at these wavelengths. The results are shown in column 5 in Table 2. Although the numbers are broadly consistent, within the uncertainties, we find that type 2 AGNs have, on average, steeper spectral indices than type 1 AGNs. This is expected because even within the chosen $f_{2}$ bins,

\footnotetext{
$\overline{13}$ We characterize the rest-frame 5-20 $\mu \mathrm{m}$ continuum as $f_{\nu} \propto \nu^{-\alpha}$, where $\alpha$ is the power-law index, $\nu$ are frequencies, and $f_{\nu}$ are flux densities, respectively.
} 
Table 2

Broadband Continuum Shape of the Observed and Nuclear Infrared Emission of AGNs

\begin{tabular}{|c|c|c|c|c|c|c|}
\hline $\begin{array}{l}\text { Class } \\
\text { (1) }\end{array}$ & $\begin{array}{l}f_{2} \\
(2)\end{array}$ & $\begin{array}{c}N \\
(3)\end{array}$ & $\begin{array}{c}\left\langle L_{X}\right\rangle \\
(4)\end{array}$ & $\begin{array}{c}\alpha_{\text {torus }} \\
(5)\end{array}$ & $\begin{array}{c}\alpha_{\text {torus }+ \text { disk }} \\
\quad(6)\end{array}$ & $\begin{array}{c}\alpha_{\text {obs }} \\
\text { (7) }\end{array}$ \\
\hline Type 1 & All & 132 & 44.34 & $1.48 \pm 0.06$ & $1.34 \pm 0.07$ & $1.22 \pm 0.07$ \\
\hline Type 1 & $<0.5$ & 106 & 44.41 & $1.40 \pm 0.08$ & $1.27 \pm 0.08$ & $1.14 \pm 0.08$ \\
\hline Type 1 & $>0.5$ & 26 & 43.74 & $1.99 \pm 0.13$ & $1.70 \pm 0.14$ & $1.58 \pm 0.11$ \\
\hline Type 2 & All & 78 & 43.49 & $1.80 \pm 0.07$ & $1.74 \pm 0.07$ & $1.47 \pm 0.08$ \\
\hline Type 2 & $<0.5$ & 22 & 43.95 & $1.61 \pm 0.13$ & $1.55 \pm 0.14$ & $1.23 \pm 0.14$ \\
\hline Type 2 & $>0.5$ & 56 & 43.46 & $1.86 \pm 0.08$ & $1.81 \pm 0.09$ & $1.52 \pm 0.10$ \\
\hline
\end{tabular}

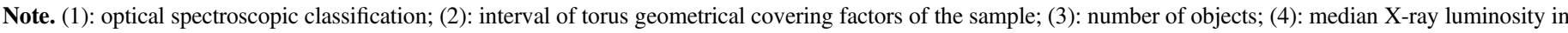

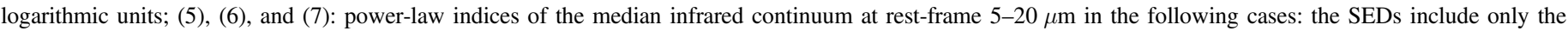

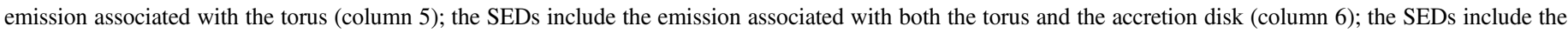
emission associated with both the torus and the accretion disk and have not been corrected for any contamination associated with the host galaxies (column 7).

the former AGN class has tori with higher covering factors overall than the latter. Clearly, at rest-frame wavelengths longer that $5 \mu \mathrm{m}$ differences still exist in the continuum emission of AGN tori, even among AGNs of the same optical class.

Although, based on the shape of the torus continuum emission, type 1 and type 2 AGNs are statistically different, there is no sharp division between the nuclear infrared SEDs of the two AGN populations. This implies that from the torus continuum emission alone, we cannot unambiguously distinguish type 1 and type 2 AGNs.

Based on our results we can conclude that the covering factor of the torus is one of the main physical parameters controlling the shape of the nuclear infrared continuum emission of AGNs. Significant differences exist in the properties of the torus emission, even among AGNs of the same optical class, implying that infrared flux-limited population studies at rest-frame wavelengths shorter than $\sim 5-6 \mu \mathrm{m}$ are not free of biases against the AGN with tori with the highest covering factors. We have shown in Section 4.2 that these objects are, on average, the most highly absorbed AGNs in $\mathrm{X}$-rays.

\subsubsection{Near-infrared Hot Dust Emission}

A broad near-infrared bump above the extrapolation of the rest-frame $>5 \mu \mathrm{m}$ continuum is clearly detected in the SEDs of our type 1 and type 2 AGNs with tori with low covering factors at rest-frame wavelengths $\sim 1-4 \mu \mathrm{m}$. The physical origin of such feature is still not clear. It could be associated with thermal radiation from hot dust in the innermost part of the torus heated by the AGN radiation field and with near sublimation temperatures (for graphite-type and silicate-type grains) or, alternatively, it might be emission from hot dust not associated with the torus (e.g in the Narrow Line Region; Edelson \& Malkan 1986; Barvainis 1987; Minezaki et al. 2004; Kishimoto et al. 2007; Schweitzer et al. 2008; Mor et al. 2009; Riffel et al. 2009; Mor \& Netzer 2012).

We have fitted the rest-frame 1-20 $\mu$ m median SEDs of our type 1 and type 2 AGNs with low torus covering factors with a two-component phenomenological model consisting of a power law and a blackbody to account for the near-infrared bump. We stress that this model is not physically motivated, nor are we claiming that the near-infrared bump originates in a separate component from the torus. Indeed, we find acceptable fits for all torus-only SEDs at rest-frame 1-20 $\mu \mathrm{m}$ with the N08 models, and after a careful visual check of the results from BayesCLUMPY, we find no evidence for additional components. The goal of our exercise is to compare the properties of the nuclear hot dust emission in type 1 and type 2 AGNs. The results of the fits are illustrated in Figure 6 (insets in the bottom plots).

The values obtained for the mean spectral indices of the midinfrared broadband continuum and blackbody temperatures are $\alpha=1.87 \pm 0.07$ and $T=1154.2 \pm 33.2 \mathrm{~K}$ for type 1 AGNs and $\alpha=1.95 \pm 0.12$ and $T=1180.1 \pm 81.2 \mathrm{~K}$ for type 2 AGNs. The best-fit temperatures indicate emission from very hot dust close to sublimation temperature. To determine the strength of the near-infrared bump we have computed its relative contribution to the integrated flux at rest-frame $2-7 \mu \mathrm{m}$. We found a contribution of $49.5 \pm 3.4 \%$ in type 1 AGNs and $41.6_{-7.8}^{+6.5} \%$ in type $2 \mathrm{AGNs}$, respectively. Clearly, not only is the near-infrared bump not exclusively detected in type 1 AGNs, but it also appears to have the same overall shape in type 1 and type 2 AGNs with tori with low covering factors.

\subsubsection{Contamination from the Accretion Disk and AGN Hosts}

So far we have analyzed nuclear infrared SEDs corrected for contamination from the extrapolated accretion disk emission and the AGN host galaxies. To compare our results with previous studies in the literature, which normally do not apply these corrections, we have analyzed the median AGN SEDs that also include the emission from the accretion disk, and the median AGN SEDs based on the cataloged photometry that include also the host galaxy emission. To do so we have followed the same approach as in Section 4.4.1, fitting the restframe 5-20 $\mu \mathrm{m}$ continuum emission with a simple power law. The results are summarized in columns 6 and 7 in Table 2. Only when we used median SEDs based on cataloged fluxes did we obtain spectral indices consistent with the typical values reported in the literature, especially for type 1 AGNs (e.g., Alonso-Herrero et al. 2006; Buchanan et al. 2006; HernánCaballero et al. 2009; Wu et al. 2009; Mullaney et al. 2011). This demonstrates that not only the emission from the accretion disk but also the stellar emission from the hosts can have a significant impact on the measured best-fit spectral indices of the infrared emission of AGN tori.

\section{DISCUSSION AND CONCLUSIONS}

Our study aims to test AGN unified models in the framework of clumpy torus models. To do so we have determined the distribution of dust covering factors of AGN tori using a large, uniformly selected, complete sample of 227 AGNs. The AGNs belong to the Bright Ultra-hard XMM-Newton Survey and have 
$z$ in the range $0.05-1.7$, and $2-10 \mathrm{keV}$ intrinsic (absorptioncorrected) luminosities between $10^{42}$ and $10^{46} \mathrm{erg} \mathrm{s}^{-1}$.

Applying data from UKIDSS, 2MASS, and WISE and a thorough SED decomposition analysis to AGN and galaxy emission, in a previous paper we determined the rest-frame 1-20 $\mu \mathrm{m}$ continuum emission associated with the torus for our sample objects. Here we modeled our nuclear infrared SEDs with the clumpy torus models of Nenkova et al. (2008a, 2008b) using the code BayesCLUMPY. This program has been particularly developed to analyze the emission of AGN tori with the Nenkova et al. (2008a, 2008b) models using a Bayesian inference approach.

The main results of our analysis can be summarized as follows.

1. Type 1, type 2, and probably also intermediate-type AGNs, are on average intrinsically different. Type 2 AGNs have tori with higher geometrical covering factors $f_{2}$ on average than type 1 AGNs. Nevertheless, the distributions of $f_{2}$ for both type 1 and type 2 AGNs are broad and there is a large overlap between the two populations. Although rare among all AGNs, we find type 1 objects with large torus covering factors (26 out of 132) and type 2 objects with small torus covering factors (22 out of 78).

2. Interestingly, type 1 and type 2 AGNs with similar levels of X-ray absorption have significantly different distributions of torus geometrical covering factors. This result also holds for X-ray unabsorbed type 1 and type 2 objects.

3. AGNs classified as Sy1.8-1.9 have a rather flat distribution of $f_{2}$ that is significantly different from those of type 1 and type 2 AGNs. Taking into account the fact that most Sy1.8-1.9s are absorbed in X-rays (12 out of 17 objects) it is unlikely that all Sy1.8-1.9 are simply ordinary type 1 AGNs caught in a low flux state during the UV/optical spectroscopic observations. Our results favor a scenario where most Sy1.8-1.9s have broad-line regions reddened by optically thin dust located either in the torus or on physical scales of the narrow-line region or the host galaxies.

4. $f_{2}$ increases with the X-ray column density, at least at $N_{H}>10^{23} \mathrm{~cm}^{-2}$, which implies that dust extinction and X-ray absorption are geometrically related and plausibly belong to the same structure, the putative dusty torus.

5. Low $f_{2}$ values are preferred at high AGN luminosities, as postulated by simple receding torus models, although for X-ray-selected type 2 AGNs, the effect is certainly small.

6. Based on our results, $f_{2}$ is one of the main physical parameters controlling the shape of the nuclear infrared emission of AGNs. Although, from the shape of the torus continuum emission, type 1 and type 2 AGNs are statistically different, there is no sharp division between the nuclear infrared SEDs of the two AGN populations. This implies that from the torus continuum emission alone, we cannot unambiguously distinguish type 1 and type 2 AGNs.

7. A broad near-infrared bump at rest-frame $\sim 1-4 \mu \mathrm{m}$ above the extrapolation of the rest-frame $>5 \mu \mathrm{m}$ infrared continuum is clearly detected in the SEDs of our type 1 and type 2 AGNs having tori with low covering factors. We find that such a spectral feature, which is often assumed to be due to hot dust in the innermost part of the torus, is not exclusively detected in type 1 AGNs and it has the same average properties in type 1 and type 2 AGNs.

Based on the results presented here, all AGNs are not intrinsically the same. This result applies not only to AGNs with different optical classifications, but also to objects of the same optical class, in agreement with predictions from clumpy torus models. The AGN radiation field can modify the covering factor of the nuclear dust obscuring the central engine, although, at least in X-ray-selected type 2 objects, the effect is rather small. Furthermore, for AGNs with X-ray absorption in the Compton-thin regime, the covering factor of the torus increases with the X-ray column density.

The reported significant differences in the torus emission, even among AGNs of the same optical class, imply that infrared flux-limited population studies at restframe wavelengths shorter than $\sim 5-6 \mu \mathrm{m}$ are not free of biases against the most highly absorbed AGNs, which we have shown are the objects with tori with the highest covering factors.

We can conclude that, the viewing angle, AGN luminosity and also $f_{2}$ determine the optical appearance of an AGN. Furthermore, $f_{2}$ controls the overall shape of the nuclear infrared continuum emission at rest-frame $\sim 1-20 \mu \mathrm{m}$. Thus, the geometrical covering factor of the dusty torus must be incorporated as a key ingredient of unification schemes.

We thank Nicolás Cardiel, R. Della Ceca, and P. Severgnini for useful comments and discussions. This work is based on observations obtained with XMM-Newton, an ESA science mission with instruments and contributions directly funded by ESA Member States and NASA. This work based on data from the Wide-field Infrared Survey Explorer, which is a joint project of the University of California, Los Angeles, and the Jet Propulsion Laboratory/California Institute of Technology, funded by the National Aeronautics and Space Administration. Funding for SDSS and SDSS-II has been provided by the Alfred P. Sloan Foundation, the Participating Institutions, the National Science Foundation, the U.S. Department of Energy, the National Aeronautics and Space Administration, the Japanese Monbukagakusho, the Max Planck Society, and the Higher Education Funding Council for England. The SDSS Web Site is http://www.sdss.org/. This work is based on observations collected at the European Organization for Astronomical Research in the Southern hemisphere, Chile, programme IDs 084.A-0828, 086.A-0612, 087.A-0447, and 088.A-0628. This work is also based on observations made with the William Herschel Telescope and its service programme - operated by the Isaac Newton Group, the Telescopio Nazionale Galileo-operated by the Centro Galileo Galilei and the Gran Telescopio de Canarias installed in the Spanish Observatorio del Roque de los Muchachos of the Instituto de Astrofísica de Canarias, in the island of La Palma. S.M., F.J.C., X.B., A.H.-C., and A.A.-H. acknowledge financial support by the Spanish Ministry of Economy and Competitiveness through grants AYA2012-31447, which is partly funded by the FEDER programme, and AYA2015-64346-C2-1-P. S.M., F.J.C. and A. A.-H. acknowledge financial support from the ARCHES project (7th Framework of the European Union, No. 313146). C.R.A. acknowledges financial support from the Marie Curie 
Intra European Fellowship within the 7th European Community Framework Programme (PIEF-GA-2012-327934). A.A.R. acknowledges financial support through the Ramón y Cajal fellowship and projects AYA2014-60476-P and ConsoliderIngenio 2010 CSD2009-00038 from the Spanish Ministry of Economy and Competitiveness. We thank the referee for the revision of the paper.

\section{REFERENCES}

Abazajian, K. N., Adelman-McCarthy, J. K., Agüeros, M. A., et al. 2009, ApJS, 182, 543

Alonso-Herrero, A., Pérez-González, P. G., Alexander, D. M., et al. 2006, ApJ, 640, 167

Alonso-Herrero, A., Quillen, A. C., Rieke, G. H., Ivanov, V. D., \& Efstathiou, A. 2003, AJ, 126, 81

Alonso-Herrero, A., Ramos Almeida, C., Mason, R., et al. 2011, ApJ, 736, 82 Antonucci, R. 1993, ARA\&A, 31, 473

Asensio Ramos, A., \& Ramos Almeida, C. 2009, ApJ, 696, 2075

Assef, R. J., Eisenhardt, P. R. M., Stern, D., et al. 2015, ApJ, 804, 27

Barvainis, R. 1987, ApJ, 320, 537

Bianchi, S., Corral, A., Panessa, F., et al. 2008, MNRAS, 385, 195

Bianchi, S., Panessa, F., Barcons, X., et al. 2012, MNRAS, 426, 3225

Brenneman, L. W., Risaliti, G., Elvis, M., \& Nardini, E. 2013, MNRAS, 429, 2662

Bruzual, G., \& Charlot, S. 2003, MNRAS, 344, 1000

Buchanan, C. L., Gallimore, J. F., O'Dea, C. P., et al. 2006, AJ, 132, 401

Buchner, J., Georgakakis, A., Nandra, K., et al. 2015, ApJ, 802, 89

Burlon, D., Ajello, M., Greiner, J., et al. 2011, ApJ, 728, 58

Caccianiga, A., Severgnini, P., Della Ceca, R., et al. 2007, A\&A, 470, 557

Caccianiga, A., Severgnini, P., Della Ceca, R., et al. 2008, A\&A, 477, 735

Calzetti, D., Armus, L., Bohlin, R. C., et al. 2000, ApJ, 533, 682

Cardelli, J. A., Clayton, G. C., \& Mathis, J. S. 1989, ApJ, 345, 245

Chabrier, G. 2003, PASP, 115, 763

Corral, A., Della Ceca, R., Caccianiga, A., et al. 2011, A\&A, 530, A42

Cutri, R. M., Severgnini, P., Della Ceca, R., et al. 2013, Explanatory Supplement to the AllWISE Data Release Products, Tech. Rep.

Cutri, R. M., Skrutskie, M. F., Van DyK, S., et al. 2003, The IRSA 2MASS All-Sky Point Source Catalog, NASA/IPAC Infrared Science Archive, http://irsa.ipac-caltech.edu/applications/Gator

Della Ceca, R., Caccianiga, A., Severgnini, P., et al. 2008, A\&A, 487, 119

Deo, R. P., Richards, G. T., Nikutta, R., et al. 2011, ApJ, 729, 108

Dullemond, C. P., \& van Bemmel, I. M. 2005, A\&A, 436, 47

Ebrero, J., Carrera, F. J., Page, M. J., et al. 2009, A\&A, 493, 55

Edelson, R. A., \& Malkan, M. A. 1986, ApJ, 308, 59

Elitzur, M. 2008, NewAR, 52, 274

Elitzur, M. 2012, ApJL, 747, L33

Fiore, F., Puccetti, S., Brusa, M., et al. 2009, ApJ, 693, 447

Gallo, L. C., MacMackin, C., Vasudevan, R., et al. 2013, MNRAS, 433, 421

Garcet, O., Gandhi, P., Gosset, E., et al. 2007, A\&A, 474, 473

Georgantopoulos, I., Rovilos, E., Akylas, A., et al. 2011, A\&A, 534, A23

González-Martín, O., Díaz-González, D., Acosta-Pulido, J. A., et al. 2014, A\&A, 567, A92

Gordon, K. D., \& Clayton, G. C. 1998, ApJ, 500, 816

Goulding, A. D., Alexander, D. M., Bauer, F. E., et al. 2012, ApJ, 755, 5

Guainazzi, M., Matt, G., \& Perola, G. C. 2005, A\&A, 444, 119

Hasinger, G., Miyaji, T., \& Schmidt, M. 2005, A\&A, 441, 417

Hernán-Caballero, A., Pérez-Fournon, I., Hatziminaoglou, E., et al. 2009, MNRAS, 395, 1695

Hönig, S. F., Kishimoto, M., Gandhi, P., et al. 2010, A\&A, 515, A23

Ichikawa, K., Packham, C., Ramos Almeida, C., et al. 2015, ApJ, 803, 57

Isobe, T., Feigelson, E. D., \& Nelson, P. I. 1986, ApJ, 306, 490

Jarrett, T. H., Chester, T., Cutri, R., et al. 2000, AJ, 119, 2498

Kishimoto, M., Hönig, S. F., Beckert, T., \& Weigelt, G. 2007, A\&A, 476, 713

Lacy, M., Ridgway, S. E., Gates, E. L., et al. 2013, ApJS, 208, 24

Lacy, M., Ridgway, S. E., Sajina, A., et al. 2015, ApJ, 802, 102

Lawrence, A., \& Elvis, M. 2010, ApJ, 714, 561

Lawrence, A., Warren, S. J., Almaini, O., et al. 1991, MNRAS, 252, 586
Lawrence, A., Warren, S. J., Almaini, O., et al. 2007, MNRAS, 379, 1599 Lira, P., Videla, L., Wu, Y., et al. 2013, ApJ, 764, 159

Lutz, D., Maiolino, R., Spoon, H. W. W., \& Moorwood, A. F. M. 2004, A\&A, 418, 465

Mainieri, V., Bergeron, J., Hasinger, G., et al. 2002, A\&A, 393, 425

Maiolino, R., Marconi, A., Salvati, M., et al. 2001, A\&A, 365, 28

Markowitz, A. G., Krumpe, M., \& Nikutta, R. 2014, MNRAS, 439, 1403

Mateos, S., Alonso-Herrero, A., Carrera, F. J., et al. 2012, MNRAS, 426, 3271

Mateos, S., Barcons, X., Carrera, F. J., et al. 2005a, A\&A, 433, 855

Mateos, S., Barcons, X., Carrera, F. J., et al. 2005b, A\&A, 444, 79

Mateos, S., Carrera, F. J., Alonso-Herrero, A., et al. 2015, MNRAS, 449, 1422

Mateos, S., Carrera, F. J., Page, M. J., et al. 2010, A\&A, 510, A35

Mateos, S., Warwick, R. S., Carrera, F. J., et al. 2008, A\&A, 492, 51

Merloni, A., Bongiorno, A., Brusa, M., et al. 2014, MNRAS, 437, 3550

Minezaki, T., Yoshii, Y., Kobayashi, Y., et al. 2004, ApJL, 600, L35

Mor, R., \& Netzer, H. 2012, MNRAS, 420, 526

Mor, R., Netzer, H., \& Elitzur, M. 2009, ApJ, 705, 298

Moran, E. C., Filippenko, A. V., \& Chornock, R. 2002, ApJL, 579, L71

Mullaney, J. R., Alexander, D. M., Goulding, A. D., \& Hickox, R. C. 2011, MNRAS, 414, 1082

Nenkova, M., Ivezić, Ž., \& Elitzur, M. 2002, ApJL, 570, L9

Nenkova, M., Sirocky, M. M., Ivezić, Ž., \& Elitzur, M. 2008a, ApJ, 685, 147

Nenkova, M., Sirocky, M. M., Nikutta, R., Ivezić, Ž., \& Elitzur, M. 2008b, ApJ, 685, 160

Netzer, H. 2015, ARA\&A, 53, 365

Nikutta, R., Elitzur, M., \& Lacy, M. 2009, ApJ, 707, 1550

Ossenkopf, V., Henning, T., \& Mathis, J. S. 1992, A\&A, 261, 567

Page, M. J., Carrera, F. J., Stevens, J. A., Ebrero, J., \& Blustin, A. J. 2011, MNRAS, 416, 2792

Page, M. J., Loaring, N. S., Dwelly, T., et al. 2006, MNRAS, 369, 156

Panessa, F., \& Bassani, L. 2002, A\&A, 394, 435

Panessa, F., Carrera, F. J., Bianchi, S., et al. 2009, MNRAS, 398, 1951

Ramos Almeida, C., Levenson, N. A., Rodríguez Espinosa, J. M., et al. 2009, ApJ, 702, 1127

Ramos Almeida, C., Levenson, N. A., Alonso-Herrero, A., et al. 2011, ApJ, 731, 92

Ramos Almeida, C., Pérez García, A. M., Acosta-Pulido, J. A., \& Rodríguez Espinosa, J. M. 2007, AJ, 134, 2006

Ricci, C., Walter, R., Courvoisier, T. J.-L., \& Paltani, S. 2011, A\&A, 532, A102

Richards, Gordon T., Strauss, M. A., Fan, X., et al. 2006, AJ, 131, 2766

Riffel, R. A., Storchi-Bergmann, T., \& McGregor, P. J. 2009, ApJ, 698, 1767

Risaliti, G., Nardini, E., Salvati, M., et al. 2011, MNRAS, 410, 1027

Risaliti, G., Salvati, M., Elvis, M., et al. 2009, MNRAS, 393, L1

Rovilos, E., Georgantopoulos, I., Akylas, A., et al. 2014, MNRAS, 438, 494

Sanders, D. B., Solomon, P. M., \& Scoville, N. Z. 1984, ApJ, 276, 182

Sazonov, S., Churazov, E., \& Krivonos, R. 2015, MNRAS, 454, 1202

Schartmann, M., Meisenheimer, K., Camenzind, M., et al. 2008, A\&A, 482, 67

Schweitzer, M., Groves, B., Netzer, H., et al. 2008, ApJ, 679, 101

Scott, A. E., Stewart, G. C., Mateos, S., et al. 2011, MNRAS, 417, 992

Severgnini, P., Caccianiga, A., Braito, V., et al. 2003, A\&A, 406, 483

Siebenmorgen, R., Heymann, F., \& Efstathiou, A. 2015, arXiv:1508.04343

Silva, L., Maiolino, R., \& Granato, G. L. 2004, MNRAS, 355, 973

Simpson, C. 2005, MNRAS, 360, 565

Stalevski, M., Fritz, J., Baes, M., Nakos, T., \& Popović, L. Č. 2012, MNRAS, 420,2756

Stern, D. 2015, ApJ, 807, 129

Strüder, L., Briel, U., Dennerl, K., et al. 2001, A\&A, 365, L18

Tozzi, P., Gilli, R., Mainieri, V., et al. 2006, A\&A, 451, 457

Treister, E., Krolik, J. H., \& Dullemond, C. 2008, ApJ, 679, 140

Tristram, K. R. W., Meisenheimer, K., Jaffe, W., et al. 2007, A\&A, 474, 837

Tristram, K. R. W., Raban, D., Meisenheimer, K., et al. 2009, A\&A, 502, 67

Ueda, Y., Akiyama, M., Hasinger, G., Miyaji, T., \& Watson, M. G. 2014, ApJ, 786, 104

Urrutia, T., Lacy, M., Spoon, H., et al. 2012, ApJ, 757, 125

Urry, C. M., \& Padovani, P. 1995, PASP, 107, 803

Winter, L. M., Mushotzky, R. F., Reynolds, C. S., \& Tueller, J. 2009, ApJ, 690,1322

Wright, E. L., Eisenhardt, P. R. M., Mainzer, A. K., et al. 2010, AJ, 140, 1868

Wu, Y., Charmandaris, V., Huang, J., Spinoglio, L., \& Tommasin, S. 2009, ApJ, 701, 658 